\title{
Monte Carlo Simulation of Ising Spins on Triangular Lattice with Different Exchange Couplings
}

\author{
Khalid Bannora*, G. Ismail*’॰ and M. Abu Zeid** \\ * Mathematics Department, Faculty of Science, Zagazig University, \\ Zagazig, Egypt \\ ** Basic Science Department, Higher Technological Institute, 10hㅡ of \\ Ramadan City, Egypt
}

The magnetization, magnetic susceptibility, internal energy and magnetic specific heat are calculated by using Monte Carlo simulation. It was applied for spin glasses (SG), $\pm J$ model, ferromagnetic (FM), and antiferromagnetic (AFM) systems on Siérpinski gasket of less than or equal to 366 Ising spins in the presence of an external magnetic field. The magnetization decreases with increasing both of the temperature and concentration of negative bonds. While on the opposite side, it increases with increasing the external magnetic field. Peaks were found in the plots of magnetic susceptibility and specific heat versus temperature or magnetic field. The internal energy increases with increasing the temperature and decreases with increasing the magnetic field. Our theoretical results are in good agreement with published experimental ones.

\section{Introduction}

Most physical systems are not isolated so they exchange energy with their environment. Since such systems are usually small in comparison to their environment, we assume that any change in the energy of the smaller system does not have a significant effect on the temperature of the larger system. Hence the larger system acts as a heat reservoir or heat bath at a fixed absolute temperature $T$ [1]. Just as there are many different types of reservoirs in the real world, there are many kinds of computer programs which can be used to simulate a reservoir. All that is required is a program which will start with any state designation and generate a succession of new designations in such a way that after many cycles the probability of any designation appearing is given by the Boltzmann distribution. 
Monte Carlo MC Simulation is an integral part of contemporary basic and applied sciences and is approaching a role equal in importance to the traditional experimental and theoretical approaches. The specific significance of the computer simulation is yielding exact information on model systems which are precisely characterized. In contrast, the information provided by analytic theory is exact only in rather rare cases, while in most other cases uncontrolled approximations are required. (MC) simulations are widely used in statistical physics [2].

Magnetization and susceptibility are calculated for FM system consisting of 366 spins situated at sites on the Siérpinski gasket by Cieplak et al. [3]. M.C. Salas-Solis et al. [4] calculated the magnetization per site for $\pm J$ Ising square lattice with size $12 \times 12$ for different anisotropies. Both the internal energy and the specific heat were calculated by Q. Zhang et al. [5]. Both magnetization and specific heat were calculated in presence of the magnetic field by W.C. Barber and D.P. Belanger [6].

In the present paper, MC on a Siérpinski gasket of 366 spins is used here instead to study the Glauber dynamics (1963) [7] for a cluster of 6 spins as in Fig.1 (top). This cluster is studied by Cieplak and Lusakowski [8] and Bannora et al. [9]. With the periodic boundary conditions the coordination number of each site is 4 , which allows studying effects of frustration. This specific Siérpinski gasket is in fact topologically equivalent to a ring of 366 spins coupled by nearest neighbor interactions. This gasket, however, is finitely ramified (Gefen et al 1980) [10] and its properties, in many respects, are reminiscent of those found in 1-dimensional systems.

The main aim for this work is to study the variety of behaviors exhibited by different distributions of the exchange interactions (SG, $\pm J, F M$ and AFM) and also the influence of both the externally applied magnetic field (B) and concentration of negative bonds $(x)$ on the magnetic and magnetothermal properties e.g. the mean value of the absolute magnetization $(\langle|M|\rangle)$, magnetic susceptibility per spin $(\chi)$, the specific heat $(C)$ and the internal energy $(\langle E\rangle)$ for these ordered (FM and AFM) and disordered (SG and $\pm J$ ) systems.

In order to explore the properties of the Ising model, we need to specify the physical properties of interest and develop a program to compute them. The layout of this paper is as follows: In section 2, we present some properties of the gasket, the model and our magnetic systems. We introduce MC simulation to 
study the dynamic parameters $(\langle|M|\rangle, \chi, C$ and $\langle E\rangle)$ in section 3. The results and discussions are presented in section 4. Finally, we summarize our conclusions in section 5 .

\section{The Model}

The Siérpinski gasket is a fractal graph which can be built with the following procedure: we begin with an equilateral triangle. The midpoints of its edges are connected, creating four triangles. The central triangle is removed (see Fig.1 bottom). The same procedure is continued for each of the new triangles down to the microscopic lattice constant. In the fractalness stage $n$, the gasket has $\frac{3}{2}\left(3^{n}+1\right)$ sites, $3^{n+1}$ edges and its side contains $2^{n}$ edges $[11,12]$. In our case, we take a system constructed by a 5 -fold division of the starting triangle of 3 spins [3]. This system consists of 366 spins and 729 exchange interactions. Periodic boundary conditions were imposed, i.e. the corner spins have been made to interact with each other. We place the system of spins into an external magnetic field $(B)$. The Hamiltonian of our systems is:

$$
E=-\sum_{\langle i j\rangle} J_{i j} S_{i} S_{j}-B \sum_{i=1}^{N_{s}} S_{i}
$$

where the first summation in (1) is over all nearest-neighbor spins and the second summation is over all spins in the lattice. The exchange constant $J_{i j}$ is a measure of the strength of the interaction between nearest-neighbor spins. In this work, we consider the following cases for the exchange interaction [13]:

(a) Spin glass (SG), where $J_{i j}$ 's are random numbers, out of which a fraction $(x)$ is negative and a fraction $(1-x)$ is positive and are generated from the Gaussian probability distribution with zero mean and unit dispersion.

(b) $\pm J$ model, binary distribution (BD), where $J_{i j}= \pm 1$, we take $J_{i j}=-1$ with probability $(x)$ and $J_{i j}=+1$ with probability $(1-x)$.

(c) Ferromagnetic (FM), $J_{i j}=+1$.

(d) Anti-ferromagnetic (AFM), $J_{i j}=-1$.

To evaluate the Hamiltonian (1), for the Siérpinski gasket, we label each spin with its site and each bond with its position. We calculate the Hamiltonian (1) term by term until all gasket sites are swept. This is a hard 
procedure to exceed this size ( $N_{s}=366$ spins) especially, there is no recurrence relation to evaluate (1) by iteration. We can take a less size such as $N_{s}=6, N_{s}=15$ and $N_{s}=42$ spins.

\section{Metropolis Monte Carlo Simulations:}

The Metropolis Monte Carlo algorithm is a well-known general method for computing the canonical equilibrium statistical expectation values by means of a weighted random sampling of the possible microstates [14]. The algorithm generates a Markov chain of configurations, $\underline{S}_{0}, \underline{S}_{1}, \ldots, \underline{S}_{n},\left(\underline{S}_{0}\right.$ is the initial configuration, $S_{i}$ is the configuration generated from $\underline{S}_{i-1}$ when we flip the $i \underline{t h}$ spin, and so on) such that the configuration $\underline{S}_{i+1}$ depends only on the immediately preceding configuration $\underline{S}_{i}$ and not on the previous history of the system [2]. The probability to get to $\underline{S}_{i+1}$ from $\underline{S}_{i}$ is given by a transition probability, $W\left(\underline{S}_{i+1} \mid \underline{S}_{i}\right)$. The transition probability is chosen such that the distribution of the states $\underline{S}_{0}, \underline{S}_{1}, \ldots, \underline{S}_{n}$ is the Boltzmann distribution,

$$
P(\underline{S}) \alpha \exp \left\{-E(\underline{S}) / k_{B} T\right\}
$$

where $k_{B}$ is the Boltzmann constant.

Suppose that we flip a single spin from $S_{i}$ to $-S_{i}$. Hence the change in energy is,

$$
\Delta E=E\left(\underline{S}_{n e w}\right)-E\left(\underline{S}_{\text {old }}\right)
$$

Because we always want to be at or near the ground state of the system [2], we should accept such a move if $\Delta E$ is less than or equal to zero. If $\Delta E$ is positive, we compute the transition probability $W=e^{-\Delta E / k_{B} T}$ and use the extraction of a uniformly distributed random number, $r \in[0,1]$. We accept or reject the new configuration [14], according to the following rules:

(i) If $r \leq W$, accept the new configuration,

(ii) If $r>W$, retain the previous configuration. 
We constructed a single-spin flipping transition probability. Once all spins have been given a chance to reverse their directions one sweep has been made. One sweep is also called one Monte Carlo step per spin (MCSS). By means of this technique, one can generate a sequence of configurations. After an opportune thermalization transient, it is possible to get the canonical equilibrium properties of the system. The Monte Carlo technique used is the single-spin flipping procedure, where in each run we discard a sufficient number of MCSS to equilibrate the system before averaging physical quantities over a number of MCSS. Data are generated with 10000 Monte Carlo steps per site after discarding the first 9000 steps. The basic thermodynamic quantities of interest are the mean absolute magnetization $(\langle|M|\rangle)$, the magnetic susceptibility per spin $(\chi)$, the mean internal energy $\langle E\rangle$ and the specific heat C. In particular, $\langle|M|\rangle, \chi$ and $C$ are computed according to the following relations:

$$
\begin{aligned}
& \langle|M|\rangle=\frac{1}{N}\left|\sum_{i=1}^{N_{s}} S_{i}\right|, \\
& \chi=\frac{1}{k_{B} T}\left(\left\langle M^{2}\right\rangle-\langle M\rangle^{2}\right), \\
& C=\frac{1}{k_{B} T^{2}}\left(\left\langle E^{2}\right\rangle-\langle E\rangle^{2}\right) .
\end{aligned}
$$

The mean of the internal energy $\langle E\rangle$ is computed using equation (1).

\section{Results and discussions:}

The dynamics of $\mathrm{SG}, \pm J, \mathrm{FM}$ and AFM systems on a Siérpinski gasket of 6, 15 and 366 Ising spins are investigated. Figure 1 shows a unit of the gasket (consists of 6 spins) and our model (consists of 366 spins). The reason for which we select the 366 lattice is that the critical temperature converges to a value by the lattice size up to 366 . Of course larger lattice would be better but since there is no recurrence relation for evaluating the Hamiltonian, we could not proceed largely (note that the number of spins is $\frac{3}{2}\left(3^{6}+1\right)=1095$ according to $\left.N_{s}=\frac{3}{2}\left(3^{n}+1\right), n=1,2,3, \ldots\right)$. So we discuss here the results of the particular gasket of 366 spins. 

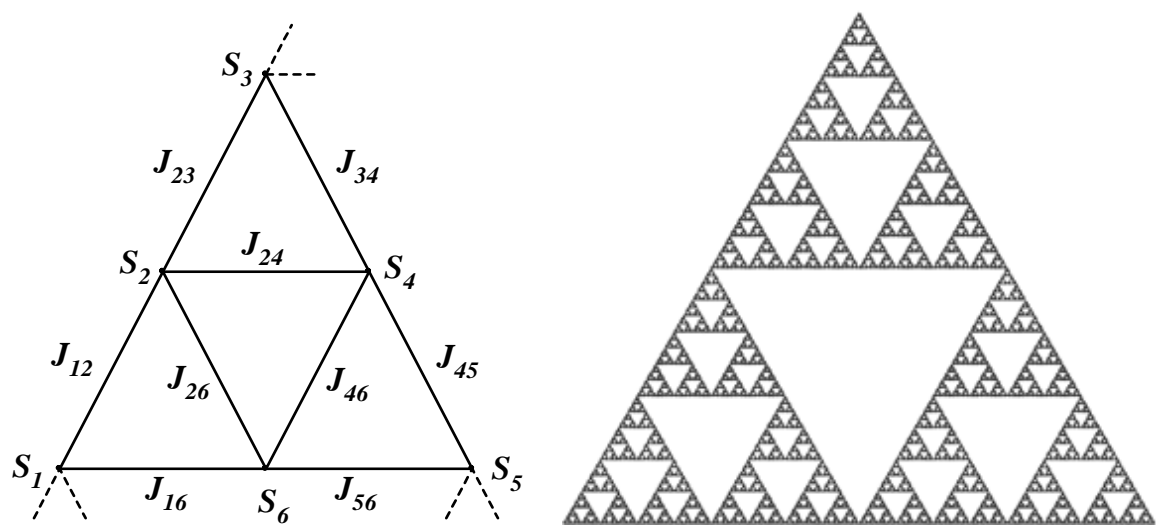

Fig. (1): A unit of the Sierpinski gasket (up) and a gasket consisting of 366 spins (down).

Figures 2(a), 2(b) and 2(c) show the dependence of the magnetization $\langle|M|\rangle$ on the temperature (T), the external magnetic field $(B)$ and the concentration of the negative bonds $(x)$, respectively.

In Fig. 2(a), $\langle|M|\rangle$ curves are concave from the right which means that $\langle|M|\rangle$ decays exponentially with increasing $T$. The curves of $\langle|M|\rangle$ are shifted up vertically when increasing $B .\langle|M|\rangle$ is saturated as $k_{B} T \leq 1.6$ for $\mathrm{FM}$ and as $k_{B} T \leq 2.6$ for $\pm J$ but for SG and AFM it decreases explicitly. We observe that $\langle|M|\rangle$ does not go to zero suddenly so, there is a second order phase transition.

Fig. 2(b), shows that in SG and $\pm J,\langle|M|\rangle$ increases logarithmically with $B$. For FM case, $\langle|M|\rangle=1$ in $0 \leq B \leq 10$ when $T=1$ and 2 but for $T=3$, $\langle|M|\rangle$ increases logarithmically on the interval $0<B \leq 2$ to saturate whatever $B>2$. At $T=4,\langle|M|\rangle$ has a rapid growth in order to reach saturation as $B \geq 5.6$. The external magnetic field $(B)$ makes the system to be more ordered. For AFM case $\langle|M|\rangle$ increases with $B$.

The mean value of the absolute magnetization $(\langle|M|\rangle)$ of SG and $\pm J$, systems decreases with increasing the concentration of the negative bonds $(x)$ as shown in Fig. 2(c). This decay of $\langle|M|\rangle$ versus $x$ is resulted by the frustration due to the high negativity. In the case of FM and AFM systems the concentration of the negative bonds $(x)$ has two fixed values which are $x=0$ and 1, respectively so $\langle|M|\rangle$ does not vary with $x$. The results are in full agreement with those of [15-21]. 


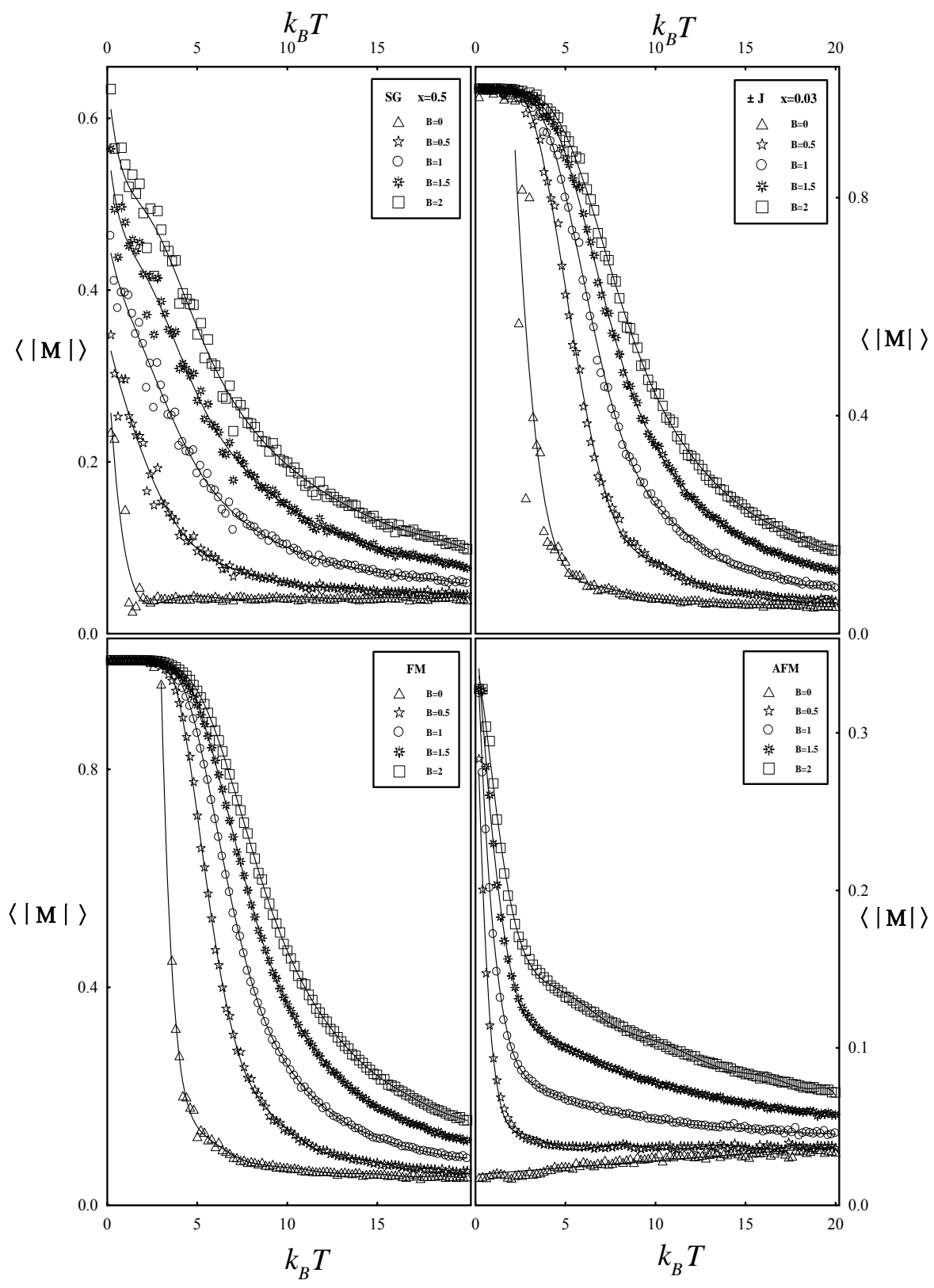




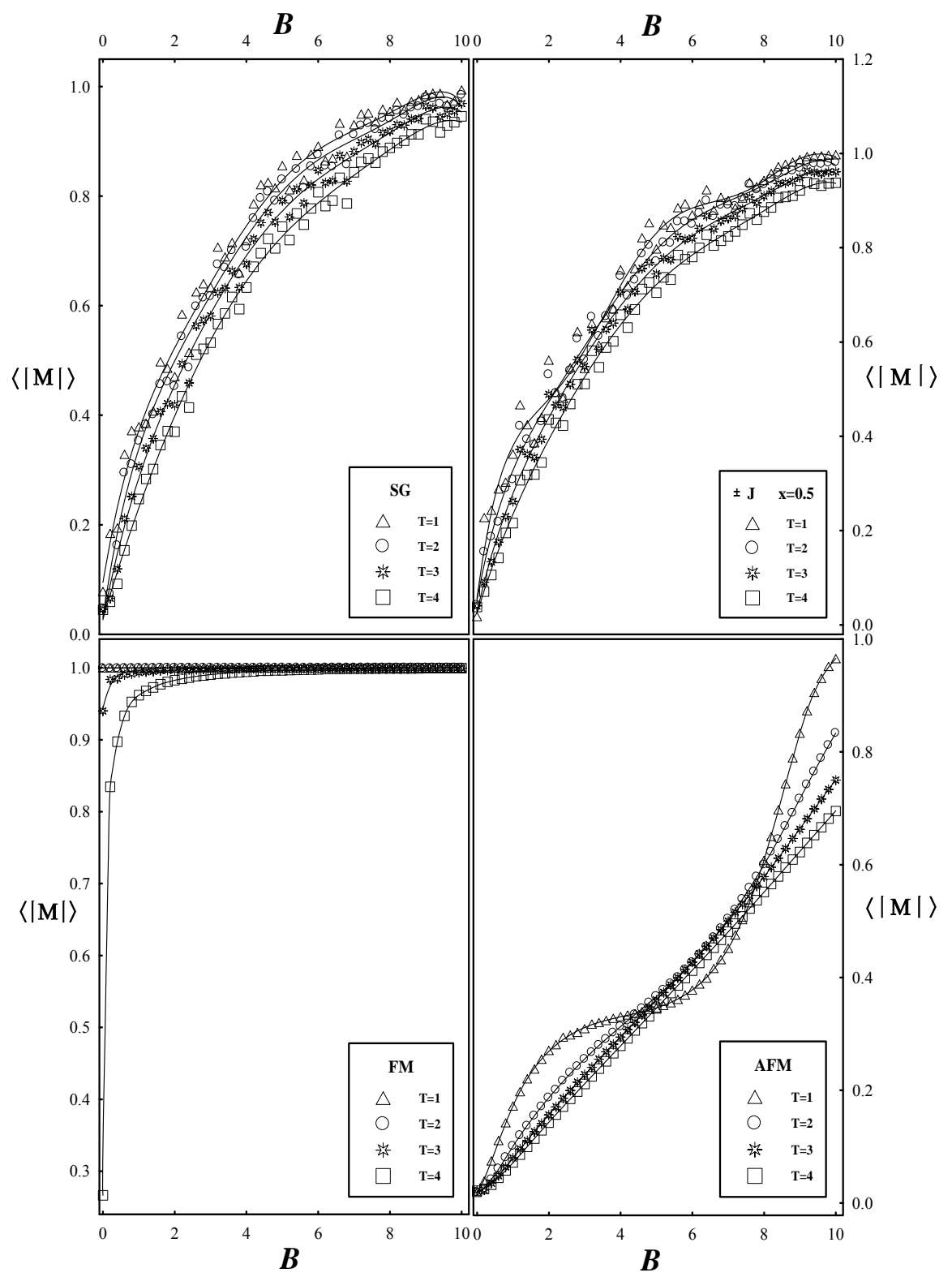




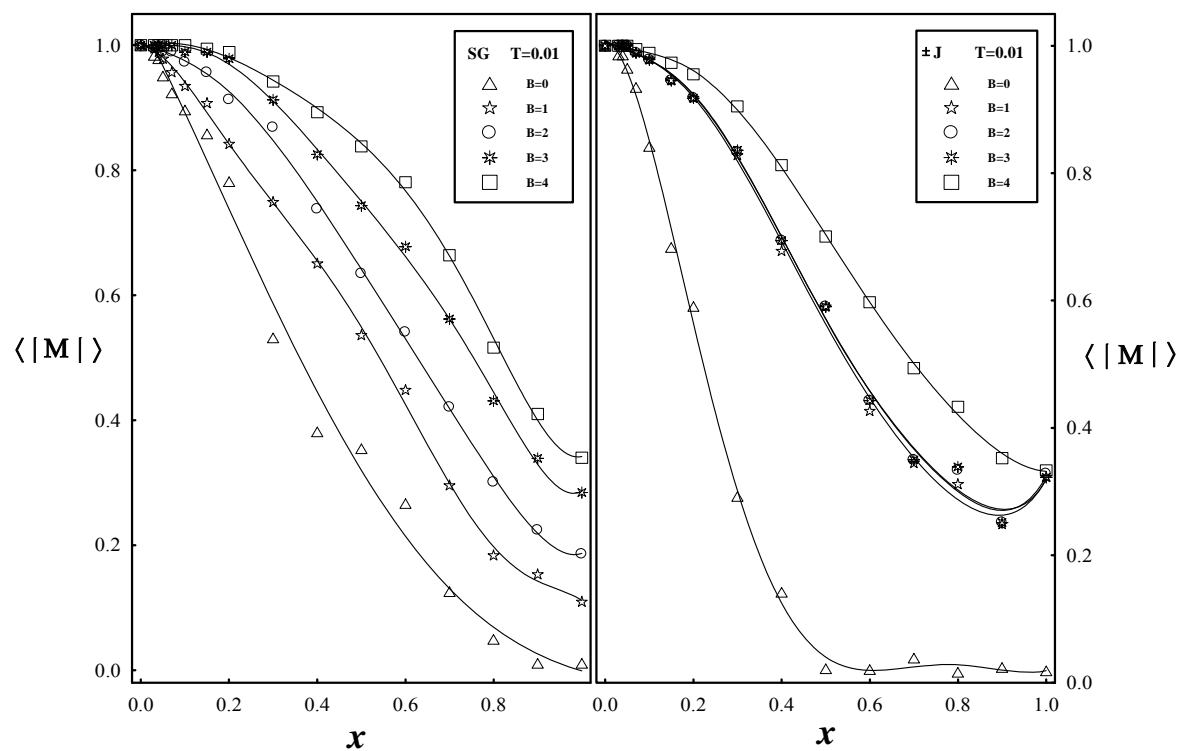

Fig. (2): [(a), (b) and (c)]: The average of absolute magnetization ( $(|M|\rangle)$ for SG, $\pm J$, FM and AFM versus temperature (T), the external magnetic field (B) and the concentration of negative bonds $(\mathrm{x})$, respectively.

The dependence of the magnetic susceptibility $(\chi)$ on the temperature and the external magnetic field are shown in Figures 3(a) and 3(b) respectively. The susceptibility curves in Fig. 3(a) have peaks for all the systems under study. For $\mathrm{SG}, \pm J$, and FM there is a sharp peak at $B=0$, but for AFM the $\chi$-curve is concave and no peak. When $B>0$ there are rounded peaks for all cases. The systems undergo a second order phase transition and the critical temperatures are located at the maxima of the magnetic susceptibility.

Fig. 3(b) shows many peaks for $\chi$ as a function of $B$ in $\pm J$ and AFM cases. While for SG there are peaks at low $B$ except at $T=0$. In FM case, $\chi$ decreases rapidly to zero.

Figures 4(a) and 4(b) show the dependence of the specific heat (C) on the temperature and the external magnetic field, respectively. In Fig. 4(a) the rounded peaks appear to explain the ability of the system to absorb heat. We notice that the critical temperatures are close to each others in SG system but they are shifted. In Fig. 4(b) the specific heat (C) has peaks for SG and $\pm J$ at low $B$ and decrease after that. In FM and AFM cases $C$ has many peaks. 


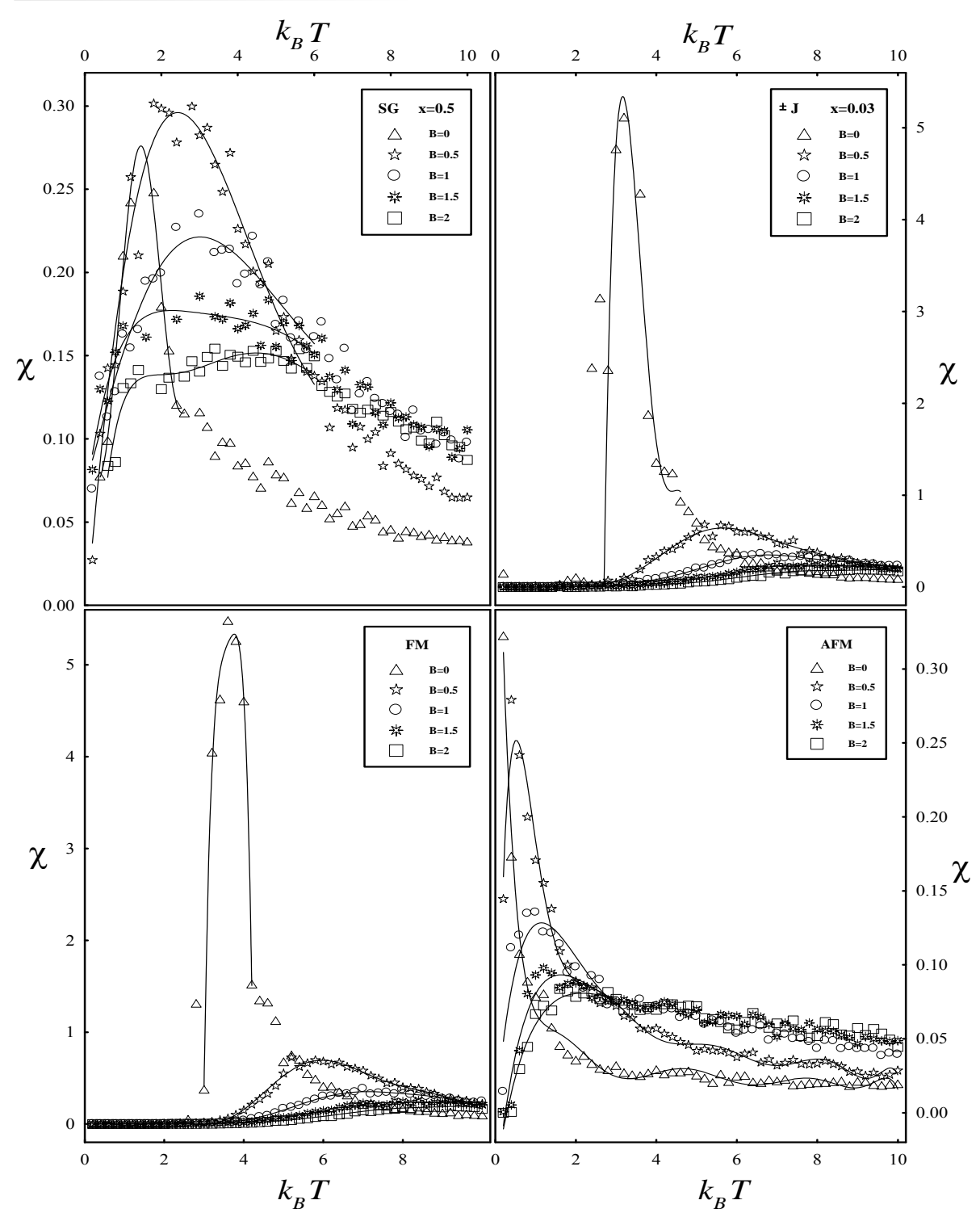

Fig.3(a) 


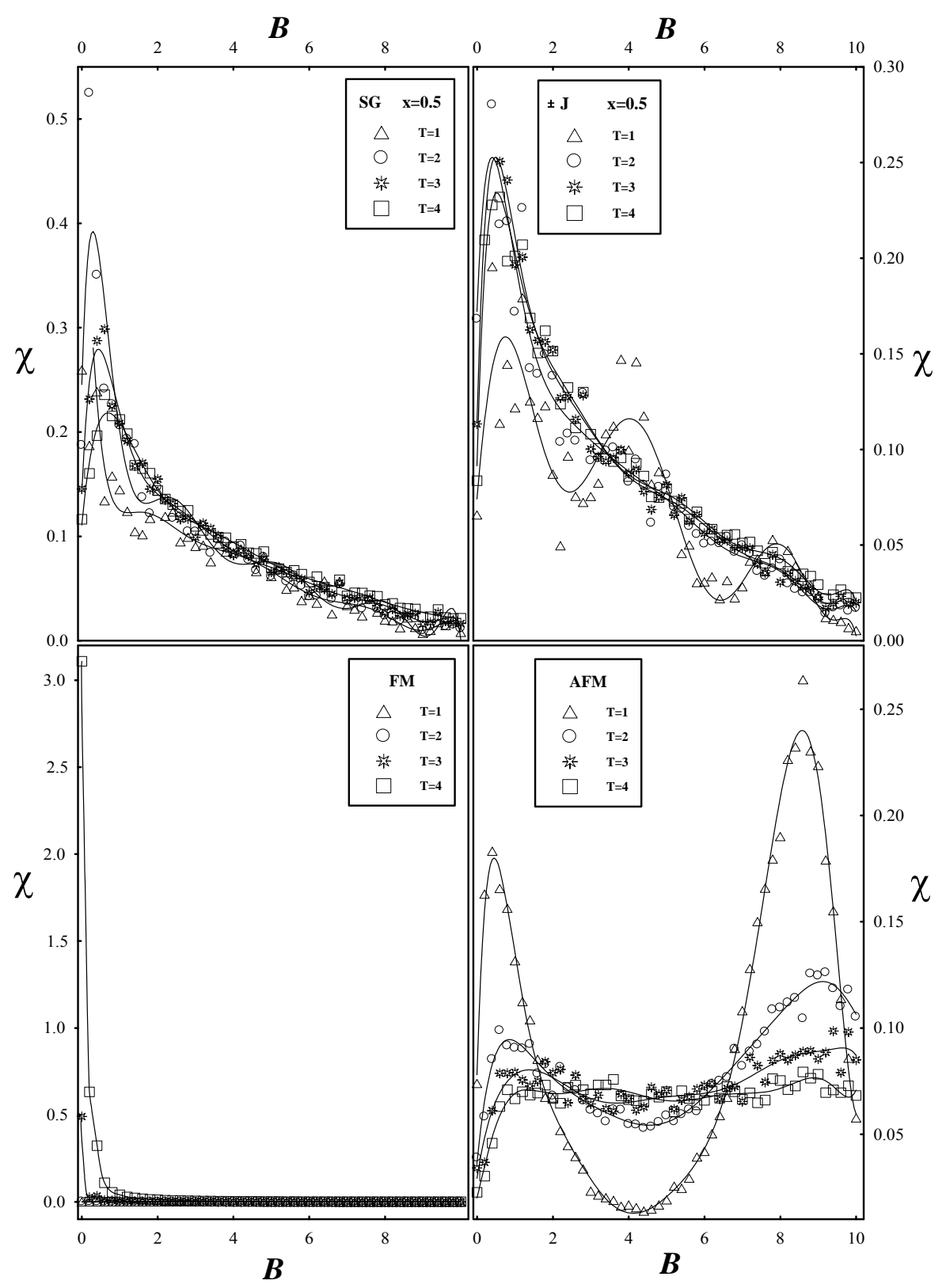

Fig.3(b) 
Fig. (3): [(a) and (b)]: The magnetic susceptibility per spin ( $\chi$ ) for $\mathrm{SG}, \pm J, \mathrm{FM}$ and AFM versus temperature $(T)$ and the external magnetic field (B), respectively. 


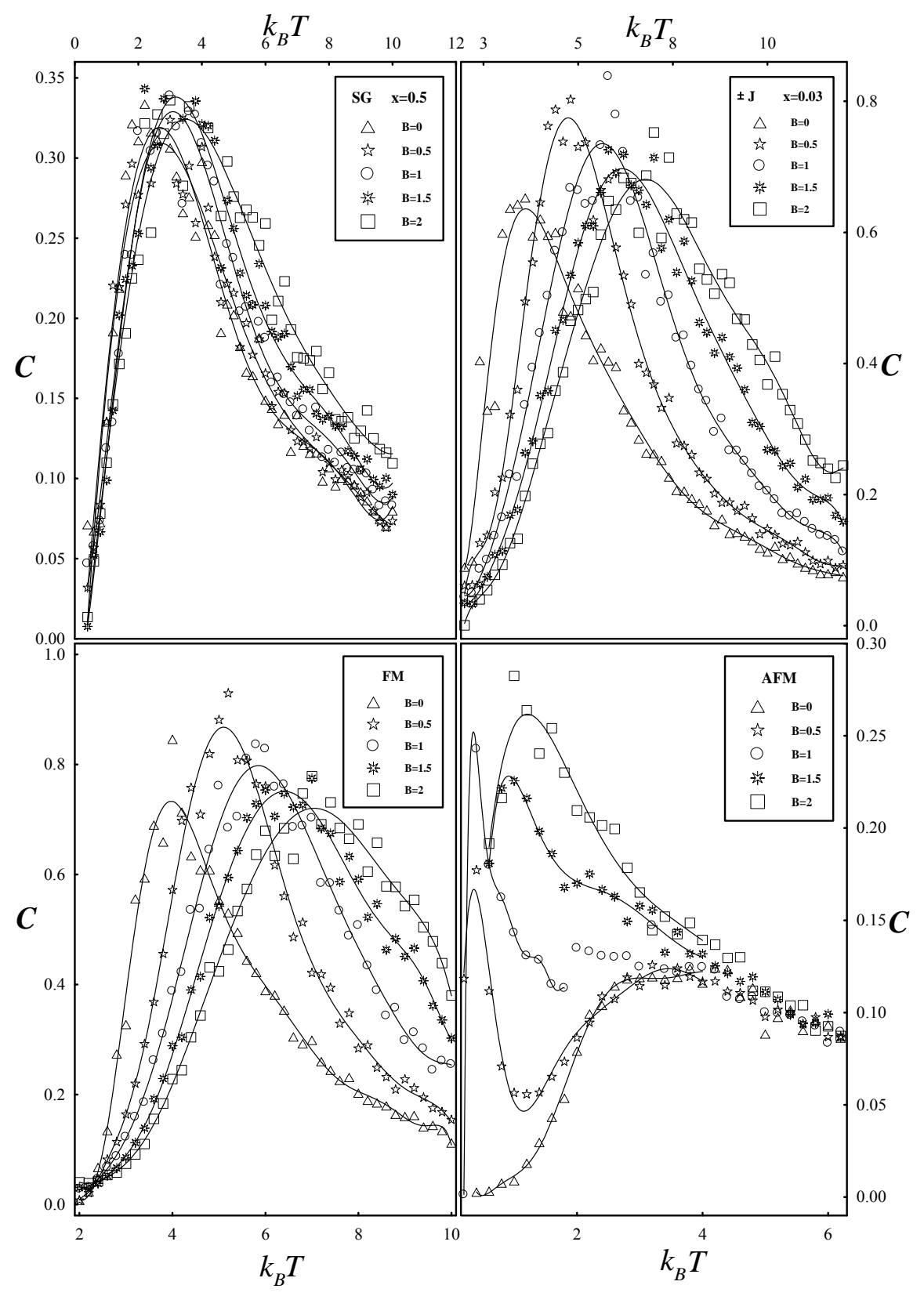

Fig.4(a) 


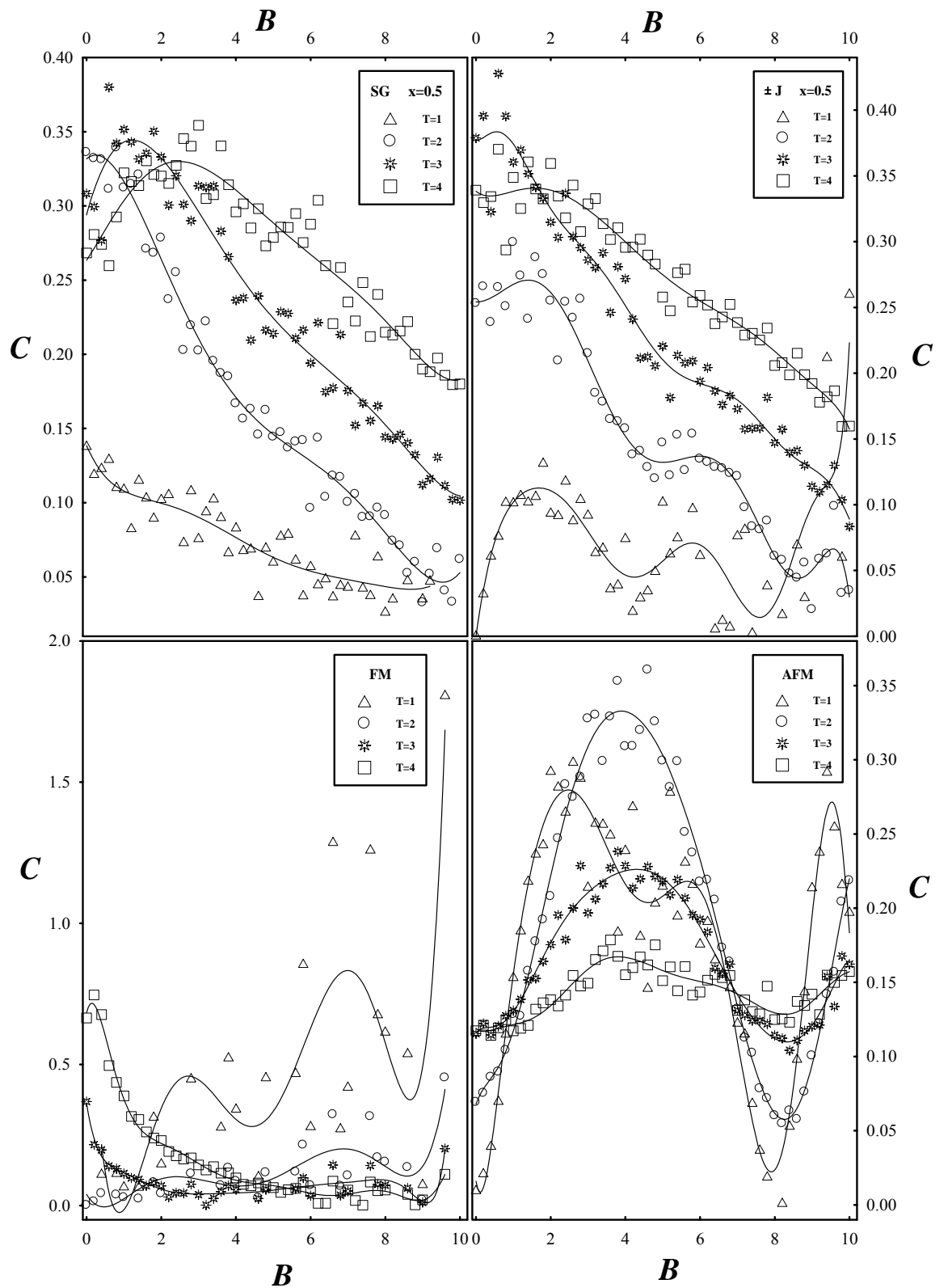

Fig. 4(b)

Fig. (4): $[(a)$ and (b)]: The specific heat $\mathrm{C}$ for $\mathrm{SG}, \pm J$, FM and $\mathrm{AFM}$ versus temperature ( $T$ ) and the external magnetic field (B), respectively. 
The dependence of the internal energy on the temperature and the external magnetic field are shown in Figures 5(a) and 5(b), respectively. The internal energy in Fig. 5(a) increases with increasing temperature for all cases. For FM and $\pm J,\langle E(T)\rangle$ is constant at low temperature then it increases $\operatorname{after} T=2.5$. We see that the energy curves are continuous. This means that the system undergoes a second order phase transition.

Fig. 5(b) shows that $\langle E\rangle$ decreases algebraically with increasing the external magnetic field $B$ for SG, $\pm J$ and AFM but linearly for FM. This means that, the discipline of the spins, in the lattice due to $B$, causes a decrease of internal energy $\langle E\rangle$.

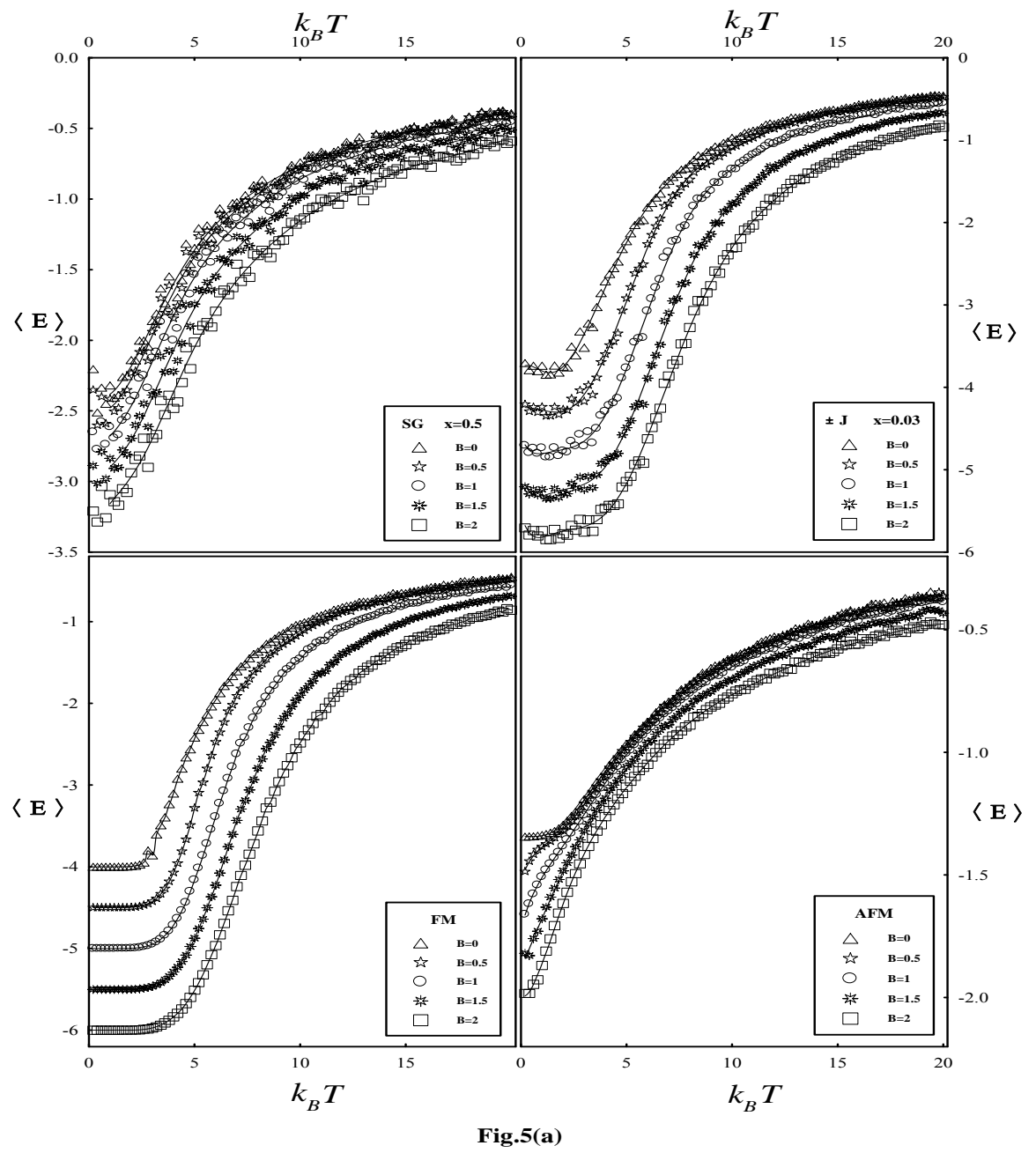




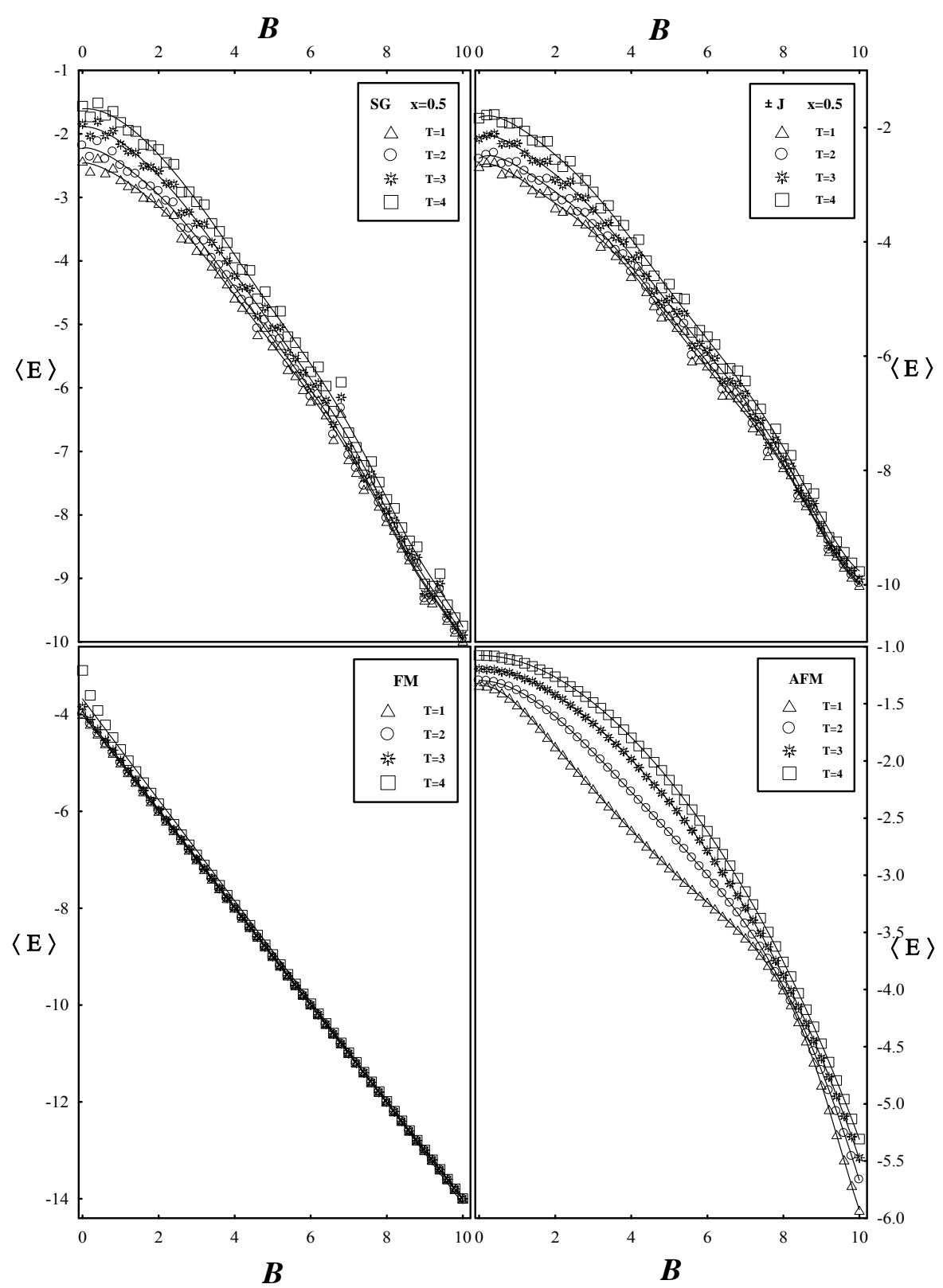

Fig.5(b)

Fig. (5): [(a) and (b)]: The internal energy $\langle E\rangle$ for SG, $\pm J$, FM and AFM versus temperature ( $T$ ) and the external magnetic field (B), respectively. 


\section{Conclusion:}

In this paper, we applied Monte Carlo (MC) simulation to calculate four dynamic parameters. Namely, the mean value of the absolute magnetization $(\langle|M|\rangle)$, magnetic susceptibility per spin, $(\chi)$ the specific heat $(C)$ and the internal energy $(\langle E\rangle)$. For two disordered; the spin glass (SG) and $( \pm J)$ model and two ordered; ferromagnetic (FM) and anti-ferromagnetic (AFM) Ising spin systems. These computations are on a Sierpinski gasket placed in an external magnetic field (B). The curves show that $\langle|M|\rangle$ does not go to zero suddenly and $\langle E\rangle$ is continuous so our model undergoes a second order phase transition. The critical temperatures are located at maxima from the curves of both $\chi$ and $C$. Now we can conclude that:

(I) The temperature (T) and the negativity concentration $(x)$ lead to the increase in the randomness and the frustration. Therefore:
(a) $\langle|M|\rangle$ decreases with $T$ or $x$,
(b) $\langle E\rangle$ increases with $T$,
(c) $\chi$ and $C$ increase with $T$ until a certain temperature at which the system undergoes a second order phase transition.

(II) The spins are aligned with the field $B$, thereby:
(a) $\langle|M|\rangle$ increases,
(b) $\langle E\rangle$ decreases
(c) $\chi$ and $C$ have many peaks at low $T$ because of the long relaxation.

\section{References:}

1. H. Gould, and J. Tobochnik, "An Introduction to Computer Simulation Methods, Applications to Physical Systems" (Part 2), Addison-Wesley, (August 30, 1995).

2. K. Binder and D. W. Heermann, "Monte Carlo simulation in statistical physics: An introduction", Springer, Berlin, (1988).

3. M. Cieplak, P. Cieplak, and M. A. Kotur, J. Phys. C 19, 4063 (1986).

4. M. C. Salas-Solis, F. Aguilera, E. E. Vogel, and J. Cartes, J. Alloys and Compounds 369, 55 (2004). 
5. Q. Zhang, G. Wei, Z. Xin, and Y. Liang, J. Magn. Magn. Mater. 280, 14(2004).

6. W. C. Barber, and D. P. Belanger, J. Magn. Magn. Mater. 226, 545 (2001).

7. R. G. Glauber, J. Math. Phys. 4, 294 (1963).

8. M. Cieplak and J. Łusakowski, J. Phys. C 19, 5253 (1986).

9. K. Bannora, G. Ismail and M. Abu Zeid, under publication in Egyptian journal of solids.

10. Y. Gefen, B. B. Mandelbrot, and A. Aharony, Phys. Rev. Lett. 45, 855 (1980).

11. R. Burioni, D. Cassi,and L. Donetti, J. Phys. A: Math. Gen. 32, 5017 (1999).

12. M. Cieplak and J. R. Banavar: Springer Series in Solid State Sciences, 54, 115 (1984).

13. J. R. Banavar and M. Cieplak: Phys. Rev. B 28, 3813 (1983).

14. A. Pluchino, G. Andronico, and A. Rapisarda: Physica A 349, 143 (2005).

15. J. Mazo-Zuluaga and J. Restrepo, Physica B 354, 20 (2004).

16. T. Iwashita, K. Uragami, K. Goto, T. Kasama, and T. Idogaki, Physica B 329, 1284 (2003).

17. T. Iwashita, K. Uragami, K. Goto, M. Arao, T. Kasama, and T. Idogaki, J. Magn. Magn. Mater. 272, 672 (2004).

18. D. V. Berkov, N. Gorn, and D. Stock: J. Magn. Magn. Mater. 272, e1281 (2004).

19. J. M. Gonzalez, O. A. Chubykalo, and R. S. Rueda, J. Magn. Magn. Mater. 203, 18 (1999).

20. R. N. Bhatt, X. Wan, M. P. Kennett, and M. Berciu, Computer Physics Communications147, 684 (2002).

21. T. Kroll, R. Klingeler, J. Geck, B. Buchner, and W. Selke, J. Magn. Magn. Mater. 290-291, 306 (2005). 